dum inflammatio est, impositam habere cum melle lenticulam: ubi ea declinavit, farinam cum ruta, vel porro, vell marrubio, donec crustae cadant: tum ervum cum melle, all irim, aut resinam terebinthinam, donec ulcus purum sit: novissime siccum linamentum." - Hier findet sich m. F. eine der oben erwihnten modernen völlig analoge Therapie, wie auch aus der Hindeutung auf die schorfhildende und secretionsheschränkende Wirkung ler verwendeten Substanzen erhellt.

\title{
VII.
}

(Aus Prof. Jassar's Klinik.)

\section{Dermatitis nach Röntgen-Strahlen}

\author{
1) r. B. PLONSKI \\ Assistent.
}

Fs hat sich in einer \%um Verhältniss der tïglich gemachten Aufnahmen zwar relativ geringen, absolut aber doch nicht kleinen Anzahl ron Fällen gezeigt, dass die Rïntgen-Strahlen, oder allgemeiner ausgedrückt, das Arbeiten mit denselben im Stande ist, auf der Haut des exponirten Kïrperteils Schädigungen hervorzurufen. Es würde zu weit führen, und liegt auch nicht im Ralimen dieser Mittheilung, alle diese Faille aus der Iitteratul anzuführen, nur so viel möge genügen, dass Gillchrist bis Anfang 1897 bereits 28 Fialle von Schädigungen der Haut durch Röntgenstrahlen zusammenstellen konnte. Bei der Neuheit des Krankheitshildes resp. des ätologischen Momentes wie auch im Interesse der Methode sellsst ist es von Wichtigkeit, derartige Fäle zur Kenntniss $\%$ bringen, da nur auf diesem Wege aus der Summe aller beobachteten Erscheinuugen sich am ehesten die Vorkehrungen zur Verhütung der Erkrankung finden lassen werden.

Am 30. Iuli stellte sich die Kutscherfrau Anna W. vor. Die Untersuchung der krätig gebauten Patientin, deren blasses Gesicht einen leidenden Ausdruck zeigte, ergab Folgendes: Aut der rechten unteren Hälfte des Abdomen fällt zunächst ein flaches Geschwür etwa von dem Lmfange einer Untertasse in die Augen. mit unregelmässigen, gezackten Rändern und dünnem, fibrinöseitrigem Belage, nach dessen Entfernung das Corium sichtbar wird. In der Peripherie dieses Geschwüres fanden sich, durch Inseln gerötheter, infiltrirter Haut getrennt, noch eine Anzahl kleinerer Ulcerationen von gleicher Beschaffenheit und eine Menge etwa kirschkerngrosser Bläschen mit eitrigem Inhalt. Die begrenzende Haut war dunkel rothbraun verfïrbt, mit zahlreichen stecknadelkopf- bis erbsengrossen flachen knötchenförmigen In- 
filtraten. Die ganze Affection, deren Breitendurchmesser 25 cm und deren Liingendurchmesser $20 \mathrm{~cm}$ betrug, voll\%or ihren Uebermang in das Gesunde durch strahlenfïrmige Ausläufer derartig verfärbter und infiltrirter Hautpartien. Dic obere Grenze befand sich vier Querfinger breit unterhalb des Leberrandes, die untere wurde durch Darmbeinkamm und durch Poupart'sches Band grehildet. Seitliche ungefähre Grenzen waren die vordere Axillarlinie und die Lina alba.

Zur Entstehung dieses /ustandes gab Patientin Folgendes an: Ende Felruar dieses .Jahres (1897) suchte sie, im letaten Drittel der Graviditait befindlich, eine gynakologische Heilanstalt auf, um sich dort wegen der bevorstehenden Entbindung Rath \%u holen, da sie auf Grund der sehr schwer verlaufenen früheren lintbindungen Befürchtungen für die kommende hegte. 'Zur Vervollstindigung der Untersuchung wurde eine Aufnalime mit XStrahlen vorgenommen. Die Inace der Beleuchtung währte eine hall,e Stunde, die Röhre befand sich nach Aussage der Patientin in einer Entfernung ron ${ }^{1 / 2}$ Meter rom Körper. A in nächsten Tage bereits verspürte Patientin an der beleuchtet gewesenen rechten Bauchseite ein heftiges Jucken und Brennen. Die Haut hatte sich in Handtellergrïsse geröthet, es entstanden Knötchen und Blasen in grosser Anzahl, letztere wurden bald eitrig, zerplatzten und an ihre Stelle traten Geschwïre. Diese Vorgänge nahmen während der letzten Zeit an Intensitait und Extensitait weiter \%u, so dass bald ein grosser Teil der rechten Bauchflache in eine heftig schmerzende (jeschwürsfïiche verwandelt war, gegen welche alle therapeutischen Massnahmen fast machtos waren. Erst nach der Mitte April erfolgten Geburt eines todten Kindes trat allmählich rom Rande her ein teilweiser lä̈ckgang des Processes ein. Nach einigen Wochen trat jedoch, ohne Veranlassung wieder eine Verschlimmerung auf, die Röthung wurde intensiver, neue Blasen- und Geschwärsbildung folgte. Da Patientin durch das lange laeiden kïrperlich sehr herunter kam und auch seelisch deprimirt wurde, wurde sie durch die Güte des behandelnden Arytes unserer Anstalt zur weiteren Behandlung überwiesen. - Hier wurde Patientin stationär mit Kleiebädern und indifferenten Salben, später je nach Erforderniss mit sublimat-Umschlägen. Wilson- und Argentumsalbe behandelt. Es wurde hierdurch innerhalb 14 Tagen eine erhebliche Verkleinerung der entründlichen und geschwürigen Stellen erreicht. Aeusserer Verhältnisse wegen musste sodann Patientin die Klinik verlassen und in die ambulatorische Behandlung übergehen, in welcher sie bis Ende Oktober verblieb: ein grosser Theil der Affection war damals unter bräunlicher Pigmentirung vernarbt: in der Mitte jedoch trotøte ein ca. kleinkinderhandgrosses und daneben ein ca. jo-P’fennigstückgrosses Geschwür jeder Behandlung. Trot\% Anwendung aller \%u Grebote stehenden einschlägigen Mittel reigten sich stets nach Alnahme des Verbandes dessen der Wunde aufliegenden Schichten mit Fiter durchtrïnkt und diese selbst filrinös-eitrigr belegt; dic angrenzenden Hautpartien waren ca. 
2 Finger breit lebhaft geröthet und leicht naissend. Patientin ent\%og sich damn der Behandlung und hat sich wie sie bei ihrer Aufang Januar h. a. erfolgten Wiederkehr angab, selbst mit Dermatol behandelt. Die geschwïrige Stelle ist cal. pflaum(rngross, flach, wenig secernirend mit leicht callösen Ränlern.

Wir haben es also mit einer ungemein heftigen und lang andauernden Hautentzündung zu thun, die nach ihrer Entstehungsart nicht anders als eine Verbrennung \%weiten und dritten Grades beyeichnet werden kann, denn dass die Beleuchtung mittels der X-Strahlen die Ursache der Erkrankung darstellt, kamn nicht be\%weifelt werden. Eine Figenthümlichkeit ler auf diese Art hervorgerufenen Verbrennungen ist das nicht unmittelbar, sonders fast stets erst längere Zeit nach Einwirkung des schädlichen Agens erfolgende Auftreten derselben. In unserem Falle machten sich die ersten Erscheinungen ca. 24 Stunden spiiter geltend, ron anderen Beobachtern wird ein noch späteres Auftreten, erst nach einigen Tagen berichtet, andrerseits kimn aber auch fist unmittelbar nach der Aufnahme der leginn der Ent\%ündung einset\%en, so hat Fuchs (D. med. Wochens(hrift 1996 S. 469 ) beobachtet, dass nach einer Beleuchtung in Intervallen wilhrend einer Stunde \%uerst Schmer\%en in den Fingergelenken entstanden und die Hand sich braun verfärbte und kur\%e Zeit darauf anschwoll; die gersamte, von den Strahlen getroffene Halutläche war mit feinen Rissen versehen, nach einer Viertelstunde bereits traten an verschiedenen Stellen Blasen auf. Ferner wird angegeben, dass arst nach wochen-, ja monatelangen Boleuchtungen die ersten lintzündungserscheinungen einset\%ten, so in der Beobachtung ron King-Toronto (Canadian Practioner, Nov. 18!)(j), wo nach \%weimonatlicher täglich 6 -10 Stunden währender Beleuchtung (es handelt sich um einen herufsmässigen S(chausteller) Rïthung, Schwellung und Blasenbildung begann. In diesem Falle trat auch ausgedehnter Haaraustali und Degeneration der Niagel ein, Erscheinungen, die auch ron anderen Beobachtern berichtest werden. Eine besondere ligigenart der Röntgen-I)ermatitis ist ferner die sehr starke Pigmentirung der nicht ulcerirten resp. wieder geheilten Hautpartien, welche von fast allen Benbachtern gesehen worden und auch in unserem falle in Firscheinung gatreten ist; hierin ähnelt sie anffïlig den durch Sounenlicht, virursachten "Verbrennungen." Fernerhin ist auffïlig die ungemein langsan vorwäts gehende Heilung der entründlichen und greschwürigen Processe; auch dies ist nicht eine besondere Figenthünlichkeit unseres Falles, sondern gleichfalls aluch von anderen Beobachtern bestätigt. So waren Sehrwald's (D. med. W. 96. s. 66j) und Drury (Brth. m. .J. 96. No. 7.) Patienten nil.h mehrmonatiger Behandlung noch nicht geheilt, ebenso war in einem neuerdings ron A postoli (Soc. Franc. d'électrothérap. Is!n, 17. Juni), veröffentlichten, dem unseren selur ähnlichen Falle, wo nach zweimaliger Beleuchtung auf der Bauchhaut eine umfangreiche resiculös- pustulöse, nachher gangränescirende Dermatitis 
entstand, eine monitelang fortgesetzte medicamentöse und chirurgische Behandlung erfolglns.

Was die eigentliche Ursache der Erkrankung ist, dariuber gehen die Meinungen noch auseinander, doch neigt man sich mehr der Ansicht \%u, dass weniger die X-Strahlen selbst, als die \%u ihrer Erzeugung nothwendigen hochgespamnten ströme als Erreger an\%uschuldigen sind, welche besonders bei grosser Nilhe der Röhre am Körper des Beleuchteten zumal bei feuchter Haut leicht in diesen selbst übergehen kïmnen. Auch Apostoli (l. (.) schreibt bei seiner Beobachitung die Dermatitis dem Umstancle \%u, dass die Crooke schen Röhren der Haut \%u sehr genälrert waren (15 cm, resp. $9 \mathrm{~cm}$ ) bei langer Dauer der Bestrahlung (40) resp. () Ninuten).

Jedenfalls ist aber auch eine besondere IDisposition der Haut erforderlich, welche das Auftreten der Ent\%ündung möglich machit, denn es werden täglich in der ganzen Welt zahhreich.. Aufnahmen gemacht, ohne dass Schädigungen der beschriehenen Art auftreten. Eine solche IDisposition muss auch bei unserer Patientin vorgelegen haben, da eine falsche Methorlik bei dor Bestrahlung augenscheinlich nicht stattgefunden hat: nach Angalue der Patientin war die Datuer der Beleuchtung eine halbe Stunde, die Röhre einen halben Meter rom Kö̈rper entfernt und wurk. eine Wiederholung nicht vorgenommen.

1)ie IDispesition kann eine bereits vorhandene sein bei Individuen mit sehr zarter Haut, wie denn auch ron einzelnen Autoren das besonders läufige Auftreten derartiger Hatentzïndungen bei Kindern betont wird, oder sie kann eine erworbene sein durch allerlei schwächende Einflüsse, Krankheiten u. s. W., sehr häufige Vornahme der Beleuchtung, woraus sich die Faille arklären lassen, bei denen lange Zoit hindurch die Anwendung der Strahlen gut vertragen und erst spät die ersten Lint\%ündungserscheinungen auftraten. In unserem Falle geht man wohl nicht fehl, wenn man die Graviditait als disponirendes Moment betrachtet. I)ie Bachhaut war stark gedehnt, in ihrer Elasticitiit herabgeset\%t, und die Patientin an und für sich in keinem hesonders guten Ernährungszustand; so war es möglich, dass eine Untersuchungsmethode, die in tausenden ron Fälen ohne jede. Folgen für den Untersuchten ist, ihr schwere, langandauernde. Schädigung verursacht hat. Es dürfte sich daher empfehlen, in jedem Falle, namentlich aber dann, wenn man eine geringe Widerstandsfähigkeit der Haut vernuthen kann, wie bei lindern schwichlichen oder betagten Individuen und Hochschwangeren für eine gehörige Ableitung des electrischen Stromes Sorge zu tragen. 